\title{
Zum 70. Geburtstag von Prof. Hans Runge
}

Am 18. April 1962 feiert H. Runge seinen 70. Geburtstag. Viele Glückwünsche von Freunden, Kollegen. Schülern und nicht zuletzt von einer großen Zahl von Patientinnen werden ihm an diesem Tag entgegengebracht. Seit 1934 Direktor der Universitäts-Frauenklinik in Heidelberg, hat Runge als einer der hervorragendsten Vertreter unseres Faches dieser Klinik Weltgeltung verschaflt. Der 18. April 1962 lordert daher zu einem Rückblick auf. Die Arbeit und das Werk Hans Runges sind nur aus dem engen Kontakt mit seinem Lehrer Schroder zu verstehen, mit dem er 1921 an der Rostocker Frauenklinik zusammentraf und der ihm die erste Anregung zu wissenschaftlicher Arbeit gab. Runge ging dann 1922 mit Schroder, als dessen Berufung auf den Kieler Lehrstuhl erfolgte. Das Wirken an der modern eingestellten Kieler Frauenklinik war ganz nach Runges Geschmack. Neben anstrengender klini-scher Tätigkeit stand in gleicher Weise wissenschaftliche Forschung. 1924 erfolgte die Habilitation und 1928 die Ernennung zum a. o. Professor. Seine didaktische Be-gabung zeigte sich schon damals: das geburtshilf liche Seminar, die Aufteilung von Studenten in kleinere Arbeitsgruppen - heute ganz modern - hat hier seine Ge-burtsstunde erlebt und wurde von den Studenten lebhaft begrüßt. 1932 erfolgte die Berufung nach Greifswald. So sehr die erste Berufung ersehnt und gefeiert wurde, Zum 70. Geburtstag von Prof. Hans Runge

267

so konnte für Runge in seinem beruflichen Werdegang Greifswald nur eine Über-gangsstation sein. Im Vordergrund stand zunächst die klinische Tätigkeit. Schon damals offenbarten sich Runges große klinische und ärztliche Qualitäten. Rei letzten Entscheidungen ve $\Gamma$ traute er viel mehr seinem intuitiven Empfinden als zweifelhaf-ten Laborbefunden. Die Arbeiten über die Cyclusanomalien wurden fortgeführt, aber schon in Greifswald tauchten die ersten Ideen über die Prophylaxe und Therapie der Thrombose und Embolie auf, und als Dissertation vergeben wurde ein Fall von vorzeitiger Plazentarlösung und Thrombopenie, die als

Rlutgerinnungsstörung be-schrieben wurde. Als junger Ordinarius wußte Runge einen Kreis von Mitarbeitern an sich zu ziehen, der bereit war, ganz nach seinen Direktiven zu arbeiten, und der ihra bei der Übersiedlung nach Heidelberg folgte. Der Ruf nach Heidelberg kam 1934, eine gleichzeitige Rerufung nach Breslau lehnte Runge ab. Die große Heidel-berger Klinik brachte naturgemäß entsprechend größere Aufgaben und weiterge-steckte Ziele. Es ist kennzeichnend für Runge, daß mit größeren Aufgaben und damit auftretenden Problemen und Schwierigkeiten seine Fähigkeiten und Talente erst in vollem Maße zur Entfaltung kamen. Ein Resignieren ist seiner ganzen Natur fremd. Zunächst wurde die Klinik nach seinen Gedankengängen und Richtlinien umgestaltet, und sodann war es ganz natürlich, daß wissenschaftliche Themen praktisch klinischen Inhaltes bearbeitet wurden. Besonders hervorzuheben sind die nach Runge benannten Zeichen der Schwangerschaftsübertragung und Überreife des Neugeborenen. Die Früherkennung des Genitalcarcinoms steht im Zusammen-hang mit seinen Arbeiten über die Redeutung der exfoliativen Cytologie. Als erster machte Runge auf die Möglichkeiten der Phasenkontrastmikroskopie für die Früherkennung des Genitalcarcinoms in der täglichen Praxis aufmerksam. Seine Stel-lungnahme über das Carcinoma in situ (sog. Oberflächencarcinom) und 
über die Ent-wicklungsdauer des Carcinoms schließen sich folgerichtig an. Die von Runge erziel-ten Heilungsergebnisse mit der elektiven Carcinomtherapie zählen zu den besten der Weltstatistik und wurden zuletzt auf dem internationalen Weltkongreß (IFGO) im September 1961 in Wien vorgetragen. Das Problem der thromboembolischen Er-krankung wurde bearbeitet. Ein nach modernsten Gesichtspunkten eingerichtetes Blutgerinnungslabor diente wissenschaftlicher Fragestellung wie klinischen Aufgaben. Als erster in Deutschland publizierte Runge seine Reobachtungen über die Defibrinierungsblutungen und gab theoretische Grundlagen und praktische Hin-weise zur Erkrankung und Rehandlung. Die Monographie «Blutung und Fluor» ist in vier Auflagen erschienen. Mehrere Handbuchartikel und über 150 wissenschaftliche Arbeiten sind publiziert worden. Als Krönung seiner Laufbahn und auf dem Höhepunkt seiner Leistung stehend, leitete Runge 1956 als Präsident der Deutschen Gesellschaft für Geburtshilfe und Gynäkologie den Heidelberger Gynäkologen-kongreß. - Eine kurze Darstellung von Runges bisherigem Leben ware unvollstän-dig, würde man nicht seine Redeutung als akademischer Lehrer hervorheben. Die Klinik in ihrer Gesamtheit wird in den Dienst des Unterrichts gestellt. Das ist kurz ausgesprochen, aber schwer zu praktizieren. Runge versteht es, Mitarbeiter heranzuziehen und ihnen verantwortliche Aufgaben zu übertragen. Er regt sie darüber hinaus zu wissenschaftlicher Arbeit an und legt strenge Maßstäbe an das, was publiziert wird.

Die vielen Besucher der Heidelberger Frauenklinik aus dem In- und Ausland werden zum Teil das alte Gebäude der Klinik abfällig betrachten. Wer jedoch weiß, wie sehr Runge sich um einen Um- oder Neubau der Klinik bernüht hat, und wer

268

Zum 70. Geburtstag von Prof. Hans Runge miterlebt hat, daß er im Jubiläumsjahr der Heidelberger Universität 1936 eine der Klinik zugedachte Spende für den Bau derKüche der damals neu erbauten Chirurgie bereitstellte, erkennt, daß Runge seine eigenen Wünsche gegenüber der Gesamt-planung zurückstellen konnte. Erst in den Jahren von 1949 bis 1951 konnte ein größerer Anbau fertiggestellt werden, der nach modernen Ansprüchen aber doch wieder bescheiden und mit sparsamsten Mitteln erbaut worden ist.

Bei den vielfältigen Anlagen und der zu leistenden Arbeit konnte man glauben, daß für persönliche Neigungen kein Raum vorhanden ist. So ist es aber nicht. Runge kann sich sehr wohl von der Klinik lösen, der Muße hingeben und Geselligkeit pfle-gen. Sein gastfreies Haus hat von jeher und bis heute vielen offengestanden.

Der faszinierenden Wirkung von Runges Persönlichkeit als Wissenschaftler, als Forscher und Arzt kann sich niemand entziehen. Prof. A. Vöge.

Buchbesprechungen - Book Reviews - Livres Nouveaux

Jores, A. und Nowakowskí, H.: Praktische Endokrinologie. G. Thieme, Stuttgart 1960. XII + 312 S., 51 Abb. DM 49.50.

Die Endokrinologie ist heute keineswegs mehr ein Sondergebiet für einige Spezialisten. Vielmehr müssen von jedem praktizierandem Arzt nicht zu geringe Kenntnisse gefordert werden. Dieses notwendige Rüstzeug will ihm dieser sorgfältig ausgearbeitete Leitfaden vermitteln. Vor allem die therapeutische Seite wírd ausführlich abgehandelt. - Daß diejenigen Endokrinopathien, mit denen sich vorwiegend der Internist zu befassen hat, umfassender besprochen werden, ist den Autoren nicht zu verargen. Die rein gynäkologisch-hormonellen Aspekte werden mehr nur gestreift. So verzichten die Autoren auf eine Darstellung der komplizierten Fragen der Diagnostik und Therapie der Zyklusstörungen. Die endokrinen Syndrome aber, 
welche Mediziner und Gynäkologe gemeinsam interessieren, werden gut dokumentiert aufgeführt. - Wir sind überzeugt, daß das Buch für viele Ärzte ein gerne konsultierter Ratgeber werden wird. H. Erb, Basel

Lehrbuch der Geburtshilfe. 13. Aufl. Hgb. von Walter Stoeckel †. VEB Gustav Fischer Verlag, Jena 1961. Mit 633 zum größten Teil farbigen Abbildungen im Text.

XVI+ 1018 Seiten. Lederin DM 56.-.

Das seit Jahrzehnten bekannte und beliebte Stoeckelsche Lehrbuch liegt nun in seiner 13.

Auflage vor. Leider war es dem Herausgeber nicht vergónnt, das Er-scheinen seines Werkes zu erleben. Er starb kurze Zeit vorher, in seinem 90. Lebens-jahr.

Als Mitarbeiter am Lehrbuch wirkten eine Reihe bekannter Gynäkologen (F. v. Míkulícz-

Radeckí, B. Ottow, E. Phílípp, L. Seitz) und der Pädiater K. Klinke mit.

Die vorliegende 13. Auflage wurde sorgfältig umgearbeitet und entspricht nun in großen Teilen den Anforderungen an ein modernes Lehrbuch. Der Text ist klar, prägnant und einprägsam. Die zahlreichen Abbildungen sind hervorragend, und die Ausstattung des Buches durch den Verlag ist sehr gut. Daß es aber «durchwegs modern» geblieben ist, wie der Herausgeber hoffte, ist leider nicht der Fall. Ab-gesehen davon, daß die Schwerpunkte in der modernen Geburtshilfe anders verteilt sind, wurden neuere Erkenntnisse in einigen Gebieten kaum beriicksichtigt

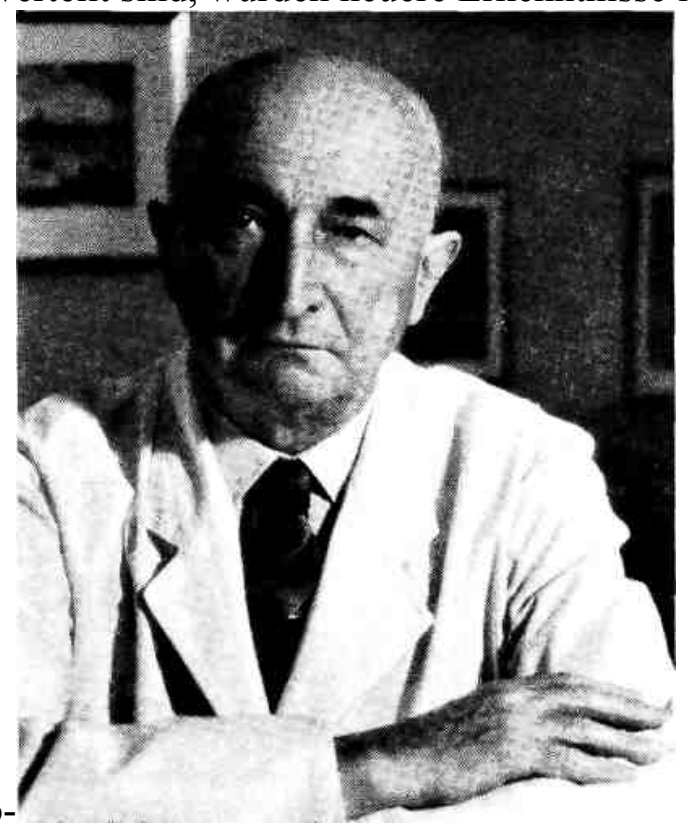

